\title{
Population Studies of Fermi LAT Galactic Sources
}

\author{
Elena Orlando*(a) (b) ${ }^{\text {Melissa Rasmussen }}{ }^{(c)}$, Andrew Strong $^{(d)}$ \\ ${ }^{(a)}$ Department of Physics, University of Trieste and National Institute of Nuclear Physics \\ via Valerio 2, 34127 Trieste, Italy \\ (b) Kavli Institute for Particle Astrophysics and Cosmology and Hansen Experimental Physics Laboratory, \\ Stanford University \\ Lomita Mall 452, Stanford, CA, U.S.A. \\ *E-mail: orlandele@gmail.com \\ (c) Utah State University (USA) \\ (d) Max Planck Institute for Extraterrestrial Physics (Germany)
}

The Fermi Large Area Telescope (LAT) has been detecting hundreds of Galactic sources, most of which are pulsars. Many Galactic sources are still undetected or unresolved due to their low flux, below the Fermi LAT sensitivity, or because of foreground and source confusion. Moreover, among the many unassociated sources, which are about one third of the detected sources, a large amount may have Galactic origin. We present our method of source population synthesis studies for characterizing the general properties of Fermi LAT Galactic gamma-ray sources and for estimating the number of Galactic sources below the Fermi LAT flux sensitivity threshold. Source density distribution and luminosity function of our Monte-Carlo simulation are constrained by the Galactic sources detected by Fermi LAT. Then, the number of unresolved sources and their contribution to the diffuse emission are estimated by our best model.

This is a long-term project on analyzing the point source catalog and on performing theoretical studies of gamma-ray sources. Apart from being interesting on its own, characterizing the general properties of detected sources also allows us to estimate the contribution to the diffuse emission from undetected and unresolved sources. In turn this helps their detection, impacting also other studies of diffuse gamma rays including studies of the interstellar emission and dark matter. Finally, it also helps in the characterization of unassociated sources. 


\section{Method}

Our analysis approach is based on the well-established method in [1,3,6]. For the Galactic source population such as pulsars, we perform a Monte Carlo study. A population is defined by three basic properties: spatial distribution, spectrum, and luminosity function. We test source space density distribution based on present models of source distributions derived from observations $[1,4]$. The space density of sources per unit luminosity $\left(\mathrm{L}_{\gamma}\right)$ and position in the Galaxy $(R, z)$ is defined as $\rho\left(\mathrm{R}, \mathrm{z}, \mathrm{L}_{\gamma}\right)$. This depends on a given luminosity function, as explained in [6]. Fluxes are then obtained from luminosities and distances. From the resulting simulated source list, we generate differential source counts defined as $\mathrm{N}\left(\mathrm{F}_{\gamma}\right)$ (sources per unit flux over the sky-area considered), with the energy dependence given by the spectral information of the source population. The simulated catalogue extends below the detection threshold (flux limit). Above the detection threshold, the simulated source counts must reproduce the real source count as detected by LAT.

\section{Preliminary Results}

\section{Detected Sources}

The aim is to characterize of the source population properties and identify unidentified sources as Galactic or extragalactic source classes through statistical analyses.

Figure 1 shows an example of the number of simulated sources for a given source density and luminosity function compared with the number of detected sources above $1 \mathrm{GeV}$ and for the inner Galaxy based on the 3FGL catalog. Simulated sources for this example are pulsar-like objects with a spatial distribution based on [4]. The plot shows source number counts above $1 \mathrm{GeV}$ in the inner Galaxy.

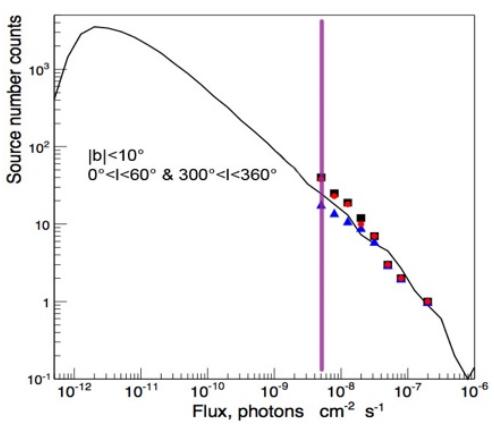

Fig. 1: An example of source number counts above 1 $\mathrm{GeV}$ in the inner Galaxy. Triangles are source counts from the 3FGL above a detection limit set at $5 \times 10^{-9}$ photons $\mathrm{cm}^{-2} \mathrm{~s}^{-1}$ above $1 \mathrm{GeV}$ (pink line). Blue triangles: Galactic identified sources. Red triangles: Galactic identified and unassociated sources. Black squares: all sources with extragalactic. Black line: simulated sources.

\section{Undetected Sources}

The aim is to estimate the number of sources below the LAT threshold and their contribution to the diffuse emission.

Figure 2 shows an example of diffuse spectral components, including the unresolved sources calculated for the model in Figure 1 (solid line), compared to 3-year LAT data. Above $8 \mathrm{GeV}$, the total component fails to account for the entire gamma-ray emission, enforcing the idea that the proposed study is needed for understanding the complete picture. We note that this model is for illustration only and it was not fit to data. 


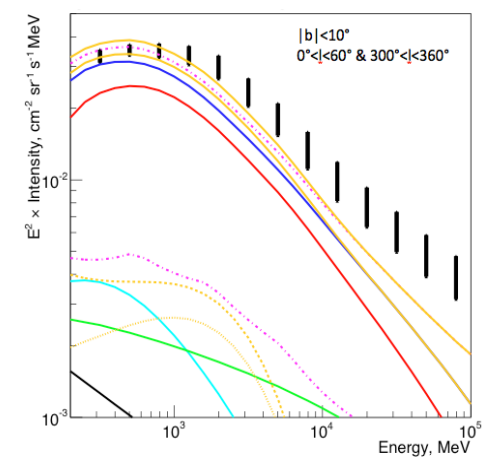

Fig. 2: An example of spectra in the inner Galaxy. We note that this model is for illustration only and it was not fit to data. Magenta dash line: 3FGL, Galactic identified and unassociated sources. Vertical black bars: Fermi-LAT data: Pass 7 Rep Clean with systematic and statistical errors included [6]. Blue line: total interstellar emission as sum of $\pi$ o-decay (red), inverse Compton (green), bremsstrahlung (cyan). Black solid line: isotropic component. Yellow lines: population contributions for a given model above (dashed upper) and below (dotted lower) threshold; total including interstellar and population contributions (solid). The interstellar model is from [2].

\section{Parameter Scan of Population Properties}

The aim is to find the best-fit parameters of the classes of sources.

As a first trial a set of models created by varying model parameters (density, minimum luminosity, luminosity index) are generated and their Log-Likelihood is obtained by fitting the pulsars sources in the 4FGL-DR2. The best-fit parameters for this preliminary run are underlined [5] and reported in the Table below.

\begin{tabular}{|llll|}
\hline $\begin{array}{l}\text { Density } \\
\text { (pulsars/kpc^3) }\end{array}$ & $\begin{array}{l}\text { Min. Luminosity } \\
\text { (photons/s) }\end{array}$ & $\begin{array}{l}\text { Index of } \\
\text { Luminosity }\end{array}$ & $\begin{array}{l}\text { Log- } \\
\text { likelihood }\end{array}$ \\
\hline 4 & $1 \times 10^{\wedge} 36$ & -2.4 & 6 \\
\hline 10 & $1 \times 10^{\wedge} 35$ & -1.8 & 66 \\
\hline 20 & $1 \times 10^{\wedge} 34$ & -1.6 & 129 \\
\hline 50 & $1 \times 10^{\wedge} 34$ & -1.8 & 152 \\
\hline 100 & $1 \times 10^{\wedge} 33$ & -1.6 & 154 \\
\hline 200 & $1 \times 10^{\wedge} 33$ & -1.8 & 141 \\
\hline
\end{tabular}

\section{Undetected Source Template}

The aim is to produce the Skymap of the model of the sources below the detection threshold as a function of energy, to be used also as a template for other studies.

\section{Conclusions}

We have presented our project on analyzing the point source catalog and on performing theoretical studies of gamma-ray sources. Preliminary results were also shown. Apart from being interesting on its own, characterizing the general properties of detected sources also allows us to estimate the contribution to the diffuse emission from undetected and unresolved sources. In turn this helps their detection, impacting also other studies of diffuse gamma rays including studies of the interstellar emission and dark matter. Finally, it also helps in the characterization of unassociated sources.

E.O. acknowledges the ASI-INAF agreement n. 2017-14-H.0 and the NASA Grant No. 80NSSC20K1558.

\section{References}

[1] Acero F. et al. 2015 ApJS 218

[2] Ackermann M. et al 2012, ApJ, 750, 3

[3] Ackermann M. et al 2013 ApJS, 209, 34

[4] Lorimer et al 2006 MNRAS, 372, 777

[5] Rasmussen M., Orlando E., Strong A.W. 2021, AAS 237 Meeting, January

[6] Strong, A.W. 2007 Ap\&SS, 309, 35 